\title{
A Longitudinal Ageing Analysis of Vocal Parameters of Singing Voice of Female Playback Singer
}

\author{
Deepali Y Loni, Shaila Subbaraman
}

\begin{abstract}
Age-related changes to the vocal structure affect the singing ability of the singer. We present a longitudinal study of vocal ageing of a female professional playback singer having more than six decades of singing span (covering singer age from 19 to 80 years). The ageing analysis is performed on six vocal parameters like - fundamental frequency (F0), vibrato, formants and spectral features like spectral roll-off and centroid. Statistical variations in these vocal parameters over the entire singing span of the singer are discussed in the paper. Significant effects noted with the ageing voice were - decrease in F0, decreased vocal range, reduction in vibrato rate, increase in vibrato extent, decrease in F2 \& F4 formants and rapid change in the spectral features. This investigation also studied the effect of ageing on singing voice quality through the measurement of singing power ratio (SPR). Increase in SPR measures was observed with ageing voice. The study of impact of vocal ageing with longitudinal data on singer identification (SID) is scare. The SID experimentation performed with 350 cappella songs covering entire singing span of the singer, showed a clear impact that change in acoustical parameters with ageing affected the performance of singer identification systems.
\end{abstract}

Keywords:. Ageing, Singing voice, Vibrato, Formants

\section{INTRODUCTION}

$\mathrm{A}_{\mathrm{g}}$ ging is a natural phenomenon in humans. Change in voice is observed at different stages over the lifespan - early childhood, teenage, youth, adulthood and in aged adults. Male adults undergo larger extent of age related voice changes especially during puberty, while female observe it around menopause [1]. The effect of change in voice is significant in professions like actors, singers, voice dubbing artists etc. The common age related signs in older singers that affect singing are loss of elasticity in lungs, reduced blood supply to larynx, stiffening of vocal folds, and changes in respiratory and cardiovascular system [2-3].

Revised Manuscript Received on January 22, 2020

* Correspondence Author

Deepali Y Loni*, Department of Electronics D. K. T. E's Textile \& Engineering Institute, Ichalkaranji, India.

Email: deepaliloni@rediffmail.com

Shaila Subbaraman, Department of Electronics Walchand College of Engineering, Sangli, India. Email: s.subbaraman@gmail.com

(C) The Authors. Published by Blue Eyes Intelligence Engineering and Sciences Publication (BEIESP). This is an open access article under the CC BY-NC-ND license (http://creativecommons.org/licenses/by-nc-nd/4.0/)
The factors contributing to changes in F0 are the physiological modifications such as changing dimension of vocal folds due to contraction of the thyroarytenoid muscle, increased mass, disorganization of the collagen fibers in the cartilages and surface irregularities [4]. These changes eventually affect the vocal fold vibration, resulting in F0 inaccuracies over the period of time.

The key acoustic feature that adds certain naturalness to the singing voice is "Vocal Vibrato". The primary origin of vibrato lies with the muscles controlling the larynx, the rhythmic variation of which in response to sub-glottic pressure leads to production of vibrato [5]. Age related modifications on larynx like - calcification of the cartilages, changes in laryngeal blood supply, thickening of laryngeal epithelium, reduced nerve conduction velocities has been reported [4]. These changes in larynx in turn affect the stability of vocal fold vibration, thus affecting the control of vibrato with age. The formants are the natural resonances of the vocal tract are also important acoustic feature of singing voice. The various degenerative changes in the vocal tract morphology changes the volume of the vocal tract [6] causing lowering of formants frequencies. This effect is greater in females than in males [1].

The hormonal fluctuations cause higher impact on voice disorders in females than in males [7]. In the survey on professional female singers conducted by Anne Elise [8], the average age for best performance reported by female singers is 30 to 50 years, after which there is decline in performance due to menopausal vocal syndrome. In another survey of Martha Elliott [9] almost 50\% female singers observed noticeable changes in the vocal system due to hormonal changes within a year after menopause.

Several experimental studies have attempted to measure the aging effect on the acoustical parameters. The experimentation on group of 72 healthy singers from age group 20-93 years for sustained production of vowel /a/, confirmed the significant decrease in F0 with age in women, while older men showed significantly higher F0 standard deviations than middle-aged men [10]. Sundberg, J. [11] observed decrease in vibrato rate with singer age and found that this decrease varies significantly at different ages among singers, with the average age around 60 year. Ulrich et al. [12] studied the effect of aging on formants of male speakers and found no significant effect on F2 or F3. Similar study by Eichhorn et al. [13] claimed effect of aging speakers on F1, F2, and F3 were vowel specific and no significant effect observed for F4. The commonly reported effects observed on vowel formants of speakers are lowering of F1 with slight to moderate decrease in F2 [14-16]. 
The purpose of this study is to focus on the effect of aging collectively on large number of singing voice parameters. Firstly it is observed that most of the aging analysis has been performed on speakers, either for sustained vowels, reading passages or recorded materials for speech related features like speech rate, sound pressure level, F0, F0 range, vowel formants, voice range profile, tremor or shakiness in the voice [1,12, 17-21]. But singing is different from speaking. Singers need responsive and precise control of number of vocal parameters like the respiratory system, laryngeal muscle activity and articulation of formants. Therefore in singing the dependence of voice parameters is more clearly evident. The effect of aging, therefore, is expected to be more pronounced on singing. Secondly, several research studies have attempted to acoustically measure the aging analysis using cross-sectional data i.e. by comparing categories of age groups from young to elderly wherein the participants (speakers or the singers) differ in the age subgroups [10, 22, $17,23]$. But the physiological differences among the participants can affect the measurement of age dependent impacts on acoustic parameters.

Longitudinal studies for aging analysis of singing voice parameters has not been explored for professional singers on a larger scale. This study experimented aging effect using longitudinal data of a professional Bollywood female playback singer. Songs of wide span are collected according to the chronological order of song recording with age of the singer and acoustic features are extracted from them. The age related variations that induce in acoustic features like - F0, vocal range, vibrato, formants and spectral features are presented in the paper over the entire singer's singing span. We also conducted voice quality assessment and experimented singer identification to find impact of aging.

\section{METHOD}

\section{A. The Female Playback Singer}

The aging analysis was carried on a professional playback singer. We selected the most respected female Indian playback singer - Lata Mangeshkar, the voice that dominated Bollywood music industry for more than five decades [24-25]. She had a long singing span career of more than six decades from 1949 to 2010. She has to her record of singing more than 25000 variety of songs in 20 different Indian languages. Known as a versatile singer, she had the ability to mould her voice and singing style according to the needs of the song at early stage in her singing career [26].

\section{B. The Singer data}

From more than thousands of songs sung by Lata Mangeshkar, we extracted nearly 350 cappella songs for performing the aging analysis. To overcome the effect of the interfering background music, we annotated manually the singing segments from the songs having no musical accompaniment. Our longitudinal data covered 62 years (1949 - 2010) of singing span of the singer that corresponds to singer age from 19 to 80 years. The distribution of songs with respect to recording year of the singer is shown in Fig 1.

The number of songs each year in the database differ depending on - availability of cappella segments from the songs and number of songs recorded by the singer each year. The length of each song in the database varies from a minimum of $3 \mathrm{sec}$ to a maximum of $20 \mathrm{sec}$. The 350 songs in our in-house developed cappella database comprised nearly 750 cappella singing segments each of $3 \mathrm{sec}$ duration, collected from commercially available CD recordings with sampling rate of $44.1 \mathrm{kHz}$.

\section{The Acoustic Analysis Procedure}

Six acoustic features namely - F0, vocal range, vibrato, formants and two spectral features were the focus of study in this paper. Consideration of these features together strongly accounted to precise description of aging effect on the vocal organs. All the features were extracted from cappella singing segments of $3 \mathrm{sec}$ duration. In the preprocessing stage, the analyzing singing voice segment were divided into frames of $25 \mathrm{~ms}$, with $15 \mathrm{~ms}$ of overlapping.

The cepstrum technique was used to isolate the glottal information from the vocal tract, for accurate estimation of F0. Fundamental frequency of each frame was computed applying center clipping autocorrelation on the cepstrum signal. Histogram and F0 contour were constructed from the F0s extracted from the frames of singing segment. The overall F0 corresponded to the highest bin of histogram. Using F0 contour, the vibrato parameters - rate and extent were extracted. The location of largest peak from the magnitude spectrum of the F0 contour described vibrato rate. The maximum and minimum deviation of the F0 contour from its mean in each vibrato period determined the vibrato extent. Formants were extracted using combination of Wavelet transform with Linear Predictive Coding (LPC) technique. The spectral peaks corresponding to the maximum amplitude were extracted as formants from each subband.

From the spectral features, we explored the spectral roll-off and centroid features whose spectral attribute are function of frequency. Spectral centroid represents spectral center of gravity i.e. it corresponds to the midpoint of spectral energy distribution [27]. It is defined as the average frequency weighted by the amplitudes present in the signal, divided by the sum of amplitudes.

$$
\text { Spectral centroid }=\frac{\sum_{k=0}^{N-1} k \cdot X(k)}{\sum_{k=0}^{W-1} X(k)}
$$

Spectral rolloff represents the frequency R below which certain fraction ' $\gamma$ ' of the spectral energy is concentrated.

$$
\sum_{k=0}^{k=R}|X(k)|^{2}=y \sum_{k=0}^{k=N-1}|X(k)|^{2}
$$

$\mathrm{R}$ is the highest frequency index which satisfies the spectral roll-off condition in Equation (2). This threshold ' $\gamma$ ' is observed to range from $70 \%$ to $95 \%$. We selected the threshold as 95\%.

All the analysis of acoustic features were done using programs developed in Matlab (version - R2013a). 


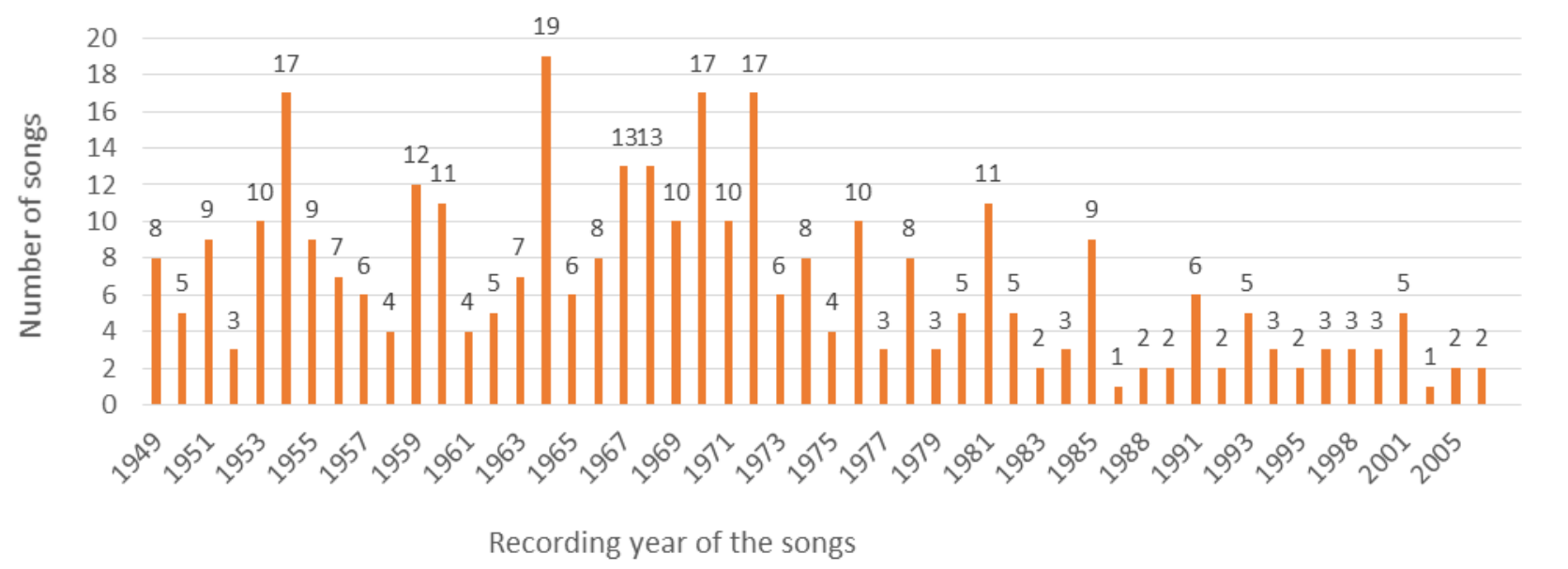

Fig. 1 Information of singer data used in the experimentation

\section{RESULTS AND DISCUSSIONS}

\section{A. Effect of Ageing on F0 of Singing Voice}

Change in F0 is the most prominent effect observed in aging voice. For F0 analysis the average F0 values of all the songs from each year were computed. The plot of average F0 values over six decades covering the entire singing years of the singer is shown in Fig. 2.

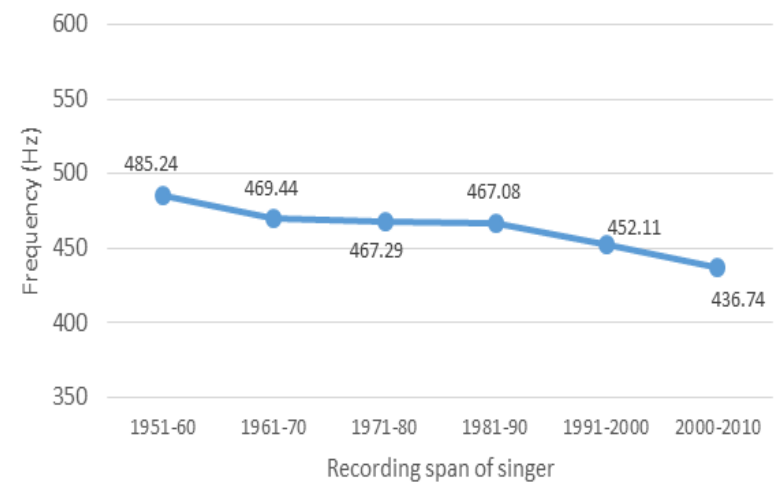

Fig. 2 Average F0 variations in the singer

The result of F0 analysis clearly indicated decrease in F0 with the age of the singer. Our longitudinal analysis result is congruent with other research studies showing decrease in F0 with aging in women [13-15, 17, 28-29]. The extent of fall when statistically measured was found to be $11.1 \%$ as compared to $22.1 \%$ reported by Jonathan et al. [15]. This clearly indicates a steady decrease in F0 of the singer as contrast to consistent decrease reported by Ulrich et al. [12] in their longitudinal study.

However our study is more consistent with Lortie et al. [10] that frequent singers show stability in F0. In the decades 60 s to 80 s, there was no significant effect of aging, revealing stable F0 characteristics of the singer. The obvious reason behind was that the singer in our study is a regular singer. It has been reported that voice of the professional singers take longer time to age, as they keep their laryngeal muscles strong by regular singing exercises [20]. The decrease in F0 in the later years of the singer may be related to hormonal effects observed in female singers.

\section{B. Effect on Vocal Range of Singer}

Vocal range describes the complete spectrum of notes that a singing voice can cover. For the analysis of vocal range, the highest and the lowest note covered by the singer were noted from the songs of each recording year. Fig. 3 shows the plot of maximum and minimum values of these notes.

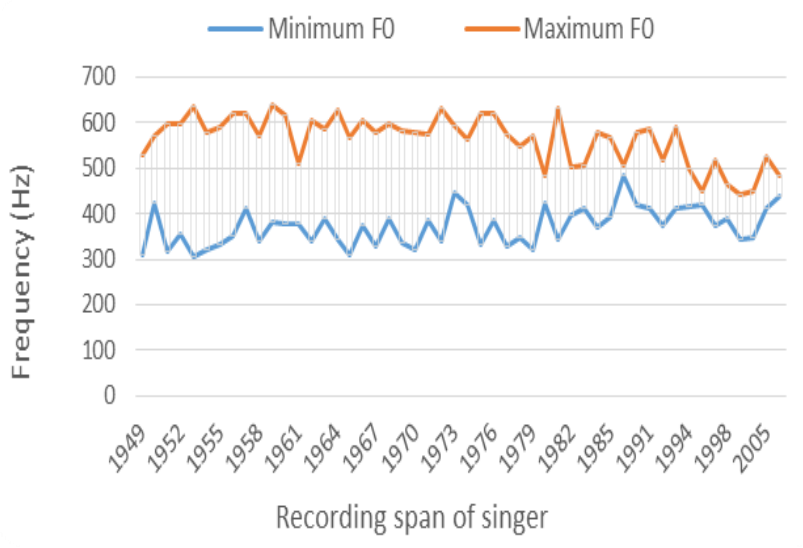

Fig. 3 Analysis of vocal range

It is observed that the F0 maximum is decreasing significantly with singer age and simultaneously there is an increasing trend observed in F0 minimum. This leads to a clear evidence of vocal range reduction with aging for the singer. The mean vocal range is observed to be substantially large ( $\cong 230.6 \mathrm{~Hz}$ ) up to 1980 s, but in the later years of singing there is noticeable reduction $(\cong 115.53 \mathrm{~Hz}$ ) by approximately $50 \%$. Similar analysis of reduction in F0 range was reported in [17] for female adults in the age group 60 -70 years. It is also reported that prolonged voice usage reduces the dynamic range of F0 [30-31].

\section{Effect of Ageing on Vibrato}

Vibrato information of the singer were extracted from the analysis of vibrato rate and extent. The plot of variation in average vibrato rate for the songs from each recording year of the singer is presented in Fig. 4.

Published By: 
From the plot we observed a gradual decrease in the vibrato rate prominently from 1990's. Reduction in vibrato rate with aging is reported in several studies [11, 32-35]. Ingo Titze et al. [36] reported reduction of $1-2 \mathrm{~Hz}$ in vibrato frequency with age. We observed a mean decrease of vibrato rate of $0.81 \mathrm{~Hz}$ in the later 30 years of singing span.

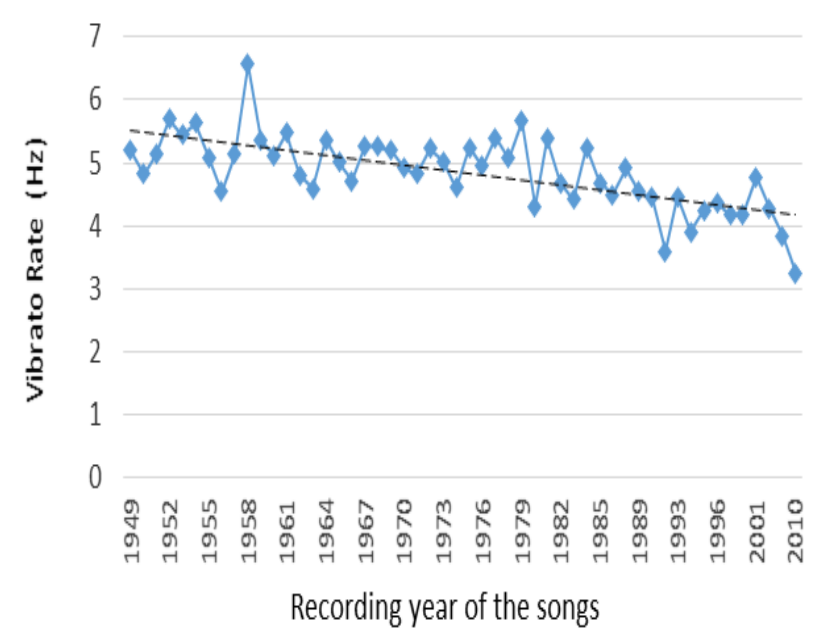

Fig. 4 Average vibrato rate of the singer

Table I presents a comparative analysis of the present study with previous studies on variations in the vibrato rate with singer aging. This table summarizes the statistical analysis and is helpful in understanding the overall trend of variations in vibrato rate with singer aging. It is observed that the rate of decrease of vibrato rate differs to some extent across the studies due to variations in the age groups of the participating singers and the span of observation. It is also observed that most of the research are based on cross-sectional studies. As the singers differ in the cross sectional studies, it is difficult to comment on the health status of the singers across the study, even though the researchers mention about selecting strong and healthy participants to volunteer for research studies.

Table I Comparative study of decrease in vibrato rate

\begin{tabular}{|c|c|c|c|}
\hline Reference & $\begin{array}{l}\text { Decrease in } \\
\text { vibrato rate }\end{array}$ & $\begin{array}{l}\text { Span of } \\
\text { observation }\end{array}$ & Participants \\
\hline [32] & $0.53 \mathrm{~Hz}$ & 30 years & $\begin{array}{l}7 \text { singers from } 30 \text { and } 60 \\
\text { age groups }\end{array}$ \\
\hline [35] & $0.9 \mathrm{~Hz}$ & 30 years & $\begin{array}{l}20 \text { singers, samples from } \\
\text { different stages of the } \\
\text { singers' careers }\end{array}$ \\
\hline [34] & $0.7 \mathrm{~Hz}$ & --- & $\begin{array}{l}21 \text { singers from } 20 \text { and } \\
65 \text { age groups }\end{array}$ \\
\hline $\begin{array}{l}\text { Present } \\
\text { study }\end{array}$ & $0.81 \mathrm{~Hz}$ & 30 years & $\begin{array}{l}\text { Longitudinal study of a } \\
\text { female singer }\end{array}$ \\
\hline
\end{tabular}

Analysis of vibrato extent of the singer is shown in Fig. 5. The analysis showed clear increase in vibrato extent with the age of the singer. Interestingly the investigation of vibrato rate and extent from Fig. 4 and 5, revealed a negative correlation between them. It is observed that in the last decade of singing span of the singer i.e. at around the singer age of 70 years, we saw increase in extent with simultaneous decrease in the rate. This analysis revealed the effect of aging on vocal chords resulting in its stretching and loss of elasticity. The change in vibrato characteristics as observed in our singer, who had been singing with vibrato her major part of singing span, gradually lost control of vibrato. On an average the vibrato extent has increased by 31.64 cent. Our longitudinal vibrato analysis supported the findings that with increasing age the singers observed decrease in vibrato rate and increased vibrato extent.

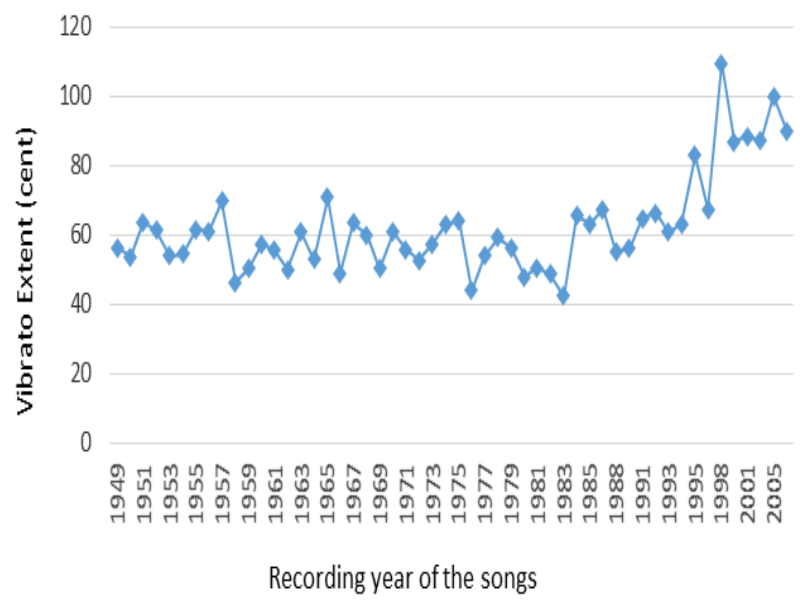

Fig. 5 Average vibrato extent of the singer

\section{Effect of Ageing on Formants}

Fig. 6 shows the variations in formant structure of five formants F1, F2, F3, F4 and F5. The aim of study was to explore the effect of aging on formants of singing voice, understand the formant variations and identify the impact on singer's formant and on higher formants.

F1
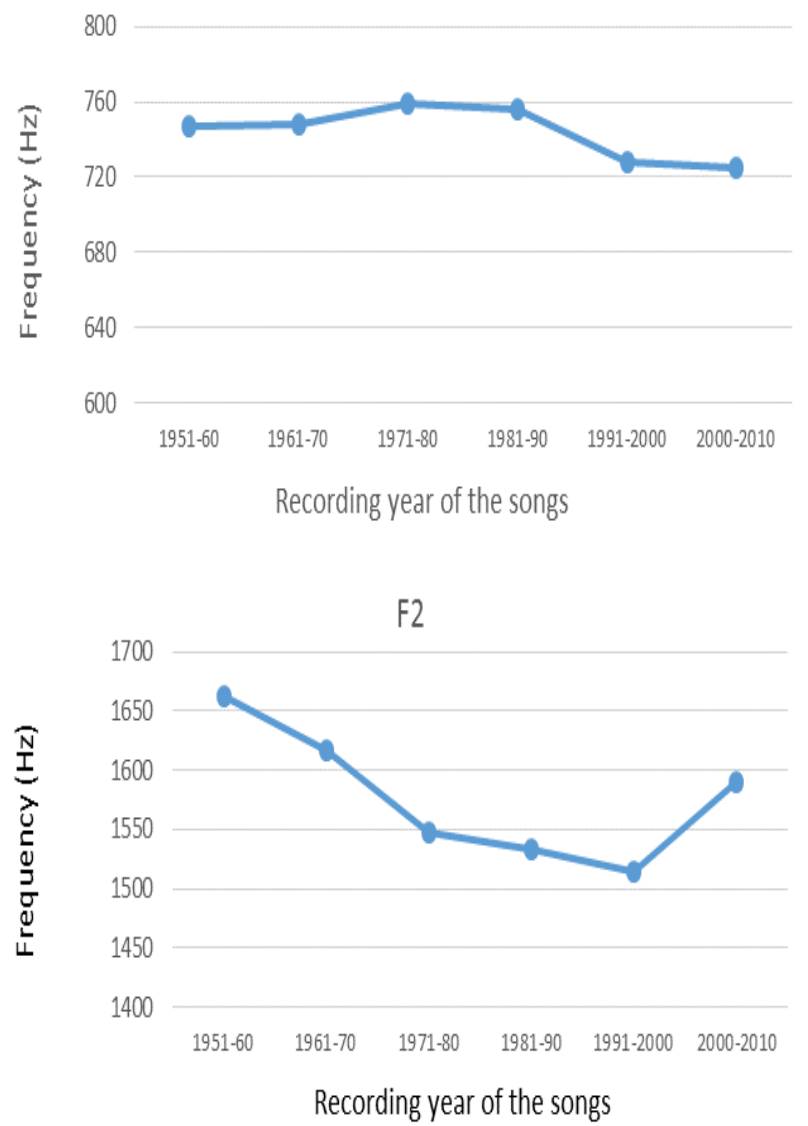
F3
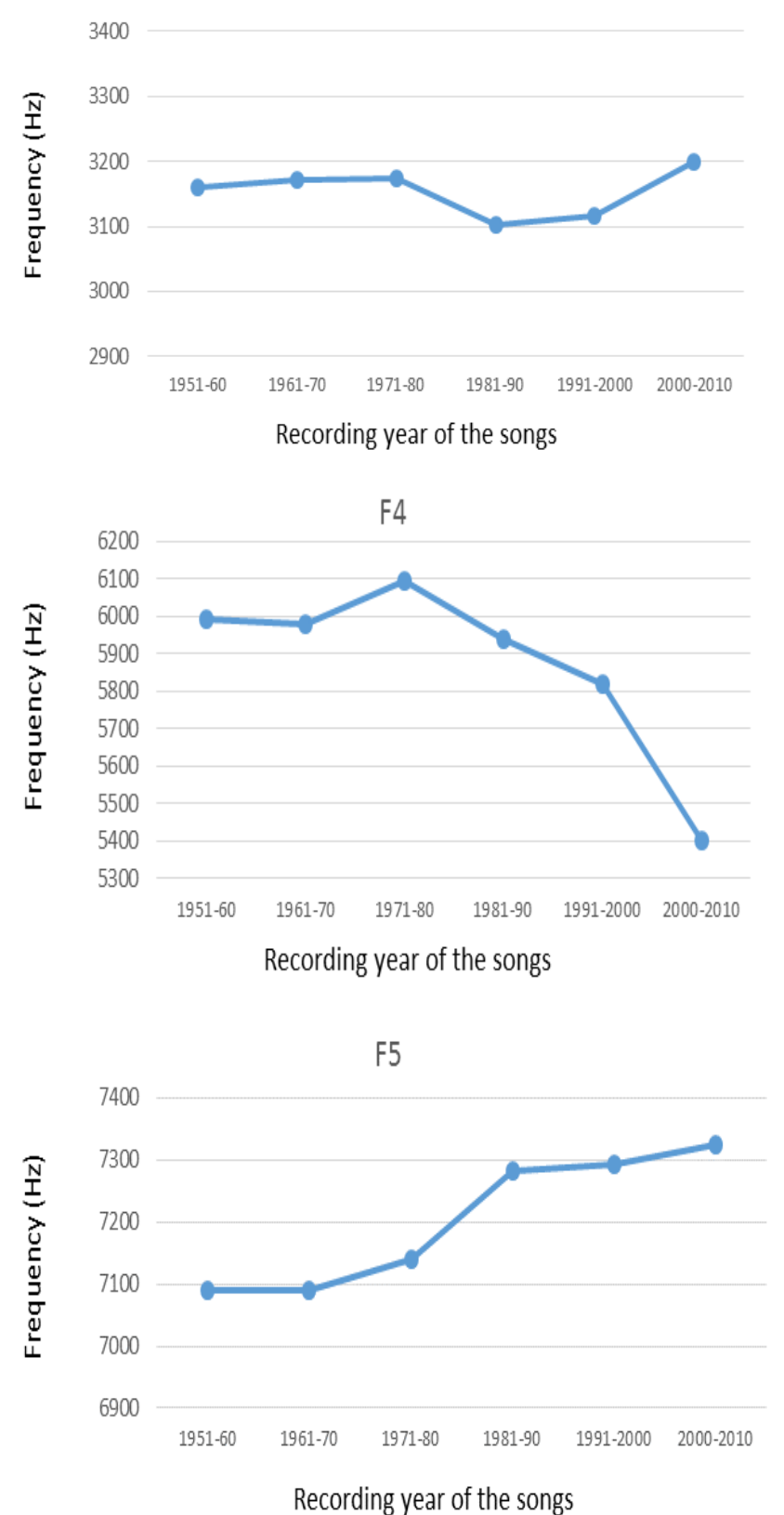

Fig. 6 Average formant frequencies of the singer over entire recording span

Analysis of formants for the singer showed clear impact on F2, F4 and F5. No significant impact was observed on formants F1 and F3 of the singer. Decrease in F2 frequency is observed with singer aging which is the most consistent finding with many research studies on formants especially for female singers [13, 15].The rate of fall in F2 from 1950s to 2000s is $8.89 \%$, although a slight increase is observed in the last decade. The center frequency of the singer's formant from the analysis of F3 was found to be in the range 3100 $3200 \mathrm{~Hz}$. The analysis indicated that maximum energy concentrated region for the singer under investigation is invariant with respect to singer aging.

Formants F4 and F5 depend on the dimension of vocal tract. Decrease in F4 can be attributed to a possible lengthening of the vocal tract, due to slight lowering of the larynx with increasing age [12]. The extent of fall in F4 $9.89 \%$ is more significant from 1950s till the last decade. Importantly, in the present study, we noticed another energy peak reinforcement in the region $7-7.4 \mathrm{KHz}$ in the $\mathrm{F} 5$ in the later years of singer age. This relates to the finding of Sang-Hyuk et al. [37] that all trained singers show another energy concentrated region from $7-8.4 \mathrm{KHz}$.

\section{E. Effect of Ageing on Spectral Features}

Fig. 7 and 8 shows the change in spectral centroid and rolloff of the singer over the entire singing span. The noticeable effect observed is rapid change in the spectral centroid and rolloff in the later age of the singer. The increase in spectral centroid indicates shift in the spectral power distribution. Correlating formant with spectral centroid, it is evident that higher spectral centroid indicated presence of high formant in the singing voice. We had observed in the formant structure analysis of the singer that in the later years of singing, high frequency formant are reinforced (especially F5). These high frequency components eventually has caused shifting of spectral centroid frequency.

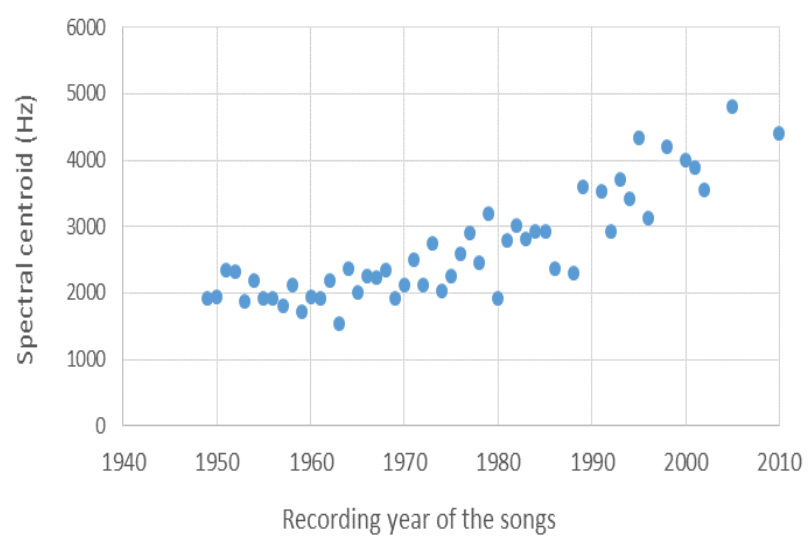

Fig. 7 Average Spectral Centroid distribution of the singer

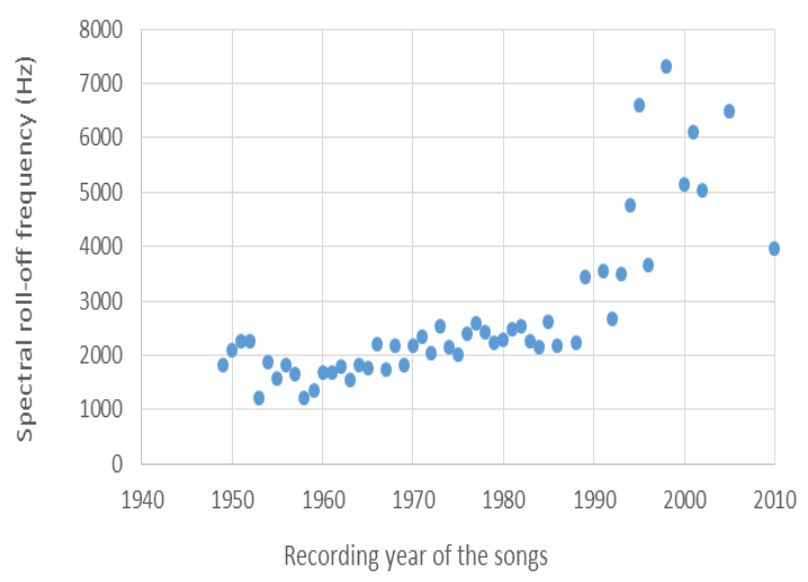

Fig. 8 Average Spectral roll-off distribution of the singer

Similar to centroid, spectral rolloff also shows rapid increase in its spectral rolloff rate. As mentioned earlier, spectral rolloff frequency indicated the frequency boundary where $95 \%$ of the total power spectrum energy reside. Rapid increase in spectral rolloff rate with the singer age clearly indicated the increased vocal effort of the singer to maintain the energy level in the singing voice.

Published By: 


\section{SINGING QUALITY ASSESSMENT - SINGING POWER RATIO}

Vocal fatigue is the frequently reported voice symptom by elderly singers. The prolonged and excessive use of voice leads to vocal fatigue that affects the quality of singing voice. Hoarseness, loss of control while singing, change in tone quality, and difficulty in sustaining duration of song are the common self-perception observations reported by the elderly singers. Voice quality, therefore, is an important indicator of the vocal health for an aging singer. The findings of Omori et al. [38] quantified singing voice quality by the measurement of singing power ratio (SPR). It is calculated using Equation (3).

$$
S P R=10 \text { abs }\left[\log 10\left(\frac{\text { formantpeak } 2 \text { to } 4 \mathrm{kHz}}{\text { formantpeak0to } 2 \mathrm{kHz}}\right)\right]
$$

SPR highlights the strength of the singers' formant cluster (F3, F4 \& F5). With the absolute value of the logarithmic ratio, lower SPR measures indicate more intense resonance in the center frequency of the singer's formant cluster. Table II presents the SPR analysis of the singer to analyze the effect of aging on the voice quality. To show a systematic evaluation of SPR analysis with the age of the singer, we divided the entire singer recording span into three parts; Recording span - I, II and III. The correlation of recording span and the singer age range is also shown in Table II. The SPR measures were extracted from all the songs from each of the recording span category. Formants were averaged separately for each year.

Table II SPR Analysis

\begin{tabular}{lll}
\hline Singer recording span & Singer age Range (years) & $\begin{array}{l}\text { Mean SPR } \pm \text { SD } \\
(\mathrm{dB})\end{array}$ \\
\hline $\begin{array}{l}1949-1962 \\
(14 \text { years })\end{array}$ & $19-32$ & $9.64 \pm 1.49$ \\
$\begin{array}{l}1963-1979 \\
(17 \text { years })\end{array}$ & $33-49$ & $10.16 \pm 1.28$ \\
$\begin{array}{l}1980-2010 \\
(31 \text { years })\end{array}$ & $50-80$ & $13.9 \pm 3.74$ \\
\hline
\end{tabular}

As evident from Table II, we observed lower SPR measures in the early singing span of the singer. SPR measure has increased with the singer age. From Recording span - I to III, we observed an increase of $6.51 \mathrm{~dB}$. But this statistical value did not reveal significant change in the SPR measure. The effect may be related to positive impact of prolonged singing span of the singer under analysis. Clearly the analysis of SPR for our female professional playback singer show slight decline in quality of singing especially for the songs in Recording span - III, an obvious impact of physiological changes in the vocal system.

\section{EFFECT OF AGEING ON SINGER IDENTIFICATION}

Singer identification (SID) is an important application of Music Information Retrieval system. Several research work on singer identification have tested the performance of the SID system for album songs, wherein the songs are recorded over a relatively short time span. The acoustic features extracted from such songs have relatively stable characteristics, resulting in higher performance of SID system. As discussed in the earlier section there are variations observed in the acoustic features if songs over large recording period are considered. These factors imposes challenges for the SID task. The aging effect on singer identification is a novel work we experimented in this paper.

Our longitudinal database of the female singer consists of nearly 350 cappella songs covering over 62 years (1949 2010) of singing span of the singer. In the first step of the experimentation, we divided the recording span of the singer in three parts and accordingly segregated the songs into different datasets - Dataset I, II and III as shown in Table III.

For the analysis of effect of aging on singer identification we used the acoustic features - vibrato, formants, spectral roll-off rate and harmonic spectral envelope (constructed from the F0 of singing voice). Considering these features along with their mean, variance, minima, maxima and peak magnitudes, we obtained 102-dimensional feature vector. Principle Component Analysis was applied on the input feature vector for dimension reduction to obtain an information - concentrated feature vector of dimension 1x60. We trained support vector machine classifier using training samples from the dataset and identified the test sample according to the largest discriminant function produced by the classifier.

We conducted the SID experimentation in three stages. A threefold cross validation approach is used to calculate the identification accuracy. In the first, we trained the classifier with songs from Dataset-I and tested the performance of the system with samples from Dataset-II and III. In the second stage, we used samples from Dataset-II and tested the performance of the system with samples from Dataset-I and III and so on in the third. Table IV shows the result of SID accuracy.

Dataset - III consists of songs in recording span that corresponds to the singers' age from 50 - 80 years. The aging effect on the acoustic features of the singing voice are more pronounced on the songs from this dataset. As observed in Table IV, the singer identification accuracy for Dataset - III is least. Also the performance is poor when the SID system is trained with Dataset - III. Better performance is observed with Dataset - II, as the acoustic parameters of the singer have reached its stable characteristics due to frequent singing. Clearly the effect of aging singing voice on singer identification revealed that the age related variations in the acoustic features affect the SID performance. Efficient SID system needs to be incorporated to compensate the variations in the acoustic features, to accurately identify the aging singers. 
Table III Classification of data for testing of SID performance

\begin{tabular}{llllc}
\hline Singer recording span & $\begin{array}{l}\text { Corresponding range of } \\
\text { singer age (years) }\end{array}$ & $\begin{array}{l}\text { Actual span of } \\
\text { available songs }\end{array}$ & Dataset label & $\begin{array}{l}\text { Number of songs } \\
\text { in the Dataset }\end{array}$ \\
\hline $\begin{array}{l}1949-1962 \text { (14 years) } \\
\begin{array}{l}1963-1979 \\
(17 \text { years })\end{array}\end{array}$ & $19-32$ & 14 years & Dataset - I & 110 \\
$1980-2010$ (31 years) & $50-80$ & 21 years & Dataset - II & 162 \\
\hline
\end{tabular}

Table IV SID performance considering ageing effect

\begin{tabular}{llll}
\hline Training data & Testing data & SID Accuracy (\%) & Overall Accuracy (\%) \\
\hline \multirow{2}{*}{ Dataset - I } & Dataset - II & 39.28 & 24.99 \\
& Dataset - III & 10.71 & 48.21 \\
\multirow{2}{*}{ Dataset - II } & Dataset - I & 57.14 & 23.21 \\
\hline \multirow{2}{*}{ Dataset - III } & Dataset - III & 39.28 & 10.71 \\
\hline
\end{tabular}

\section{CONCLUSION}

We report longitudinal study of aging analysis of a professional female playback singer covering 62 years of her singing span. For the singer under analysis notable changes in the acoustical parameters with aging were observed especially in the last decade of singer's singing span i.e. for the singer age from 65 to 80 years. Also the singing power ratio measure provide the empirical evidence of aging affecting the singing voice quality. The SID test performed with the large singing voice corpus of the singer also showed decline in performance with singer aging.

As the extent and speed of the vocal aging vary from singer to singer, it is difficult to generalize the aging effect variations among the singers. It entirely depends on the vocal health maintained by the singer and voice usage through prolonged singing. For the professional playback singer, Lata Mangeshkar, we observed a large span of nearly 60 years, wherein the acoustical parameters are relatively revealing expected performance. Our experimentation statistically proved why the singer had ruled the Bollywood music industry for a long duration and justified the title bestowed to her 'The Nightingale of India'.

\section{REFERENCES}

1. Schötz S (2007) Acoustic Analysis of Adult Speaker Age. Speaker Classification I, Springer Berlin/Heidelberg. 88-107. doi: 10.1007/978-3-540-74200-5_5

2. Butler A, Lind V, Van Weelden K (2001) Research on the Aging Voice: Strategies and Techniques for Healthy Choral Singing. Journal of the Phenomenon of Singing 3:42-50.

3. Vaughn S (2001) A Singer's Guide to Vocal Care. Journal of Singing. 57 (3): 53-60

4. Tanya L. Eadie (2000) Characteristics of the aging female voice Caracteristiques de la voix feminine vieillissante. Journal of Speech-Language Pathology And Audiology. 24(4):162 - 179.

5. N. Fletcher (2001) Vibrato in music. Acoust. Australia. 29(3):97-102.

6. Yan N, Ng M, Man ML, Hin TT (2013) Vocal tract dimensional characteristics of professional male and female singers with different types of singing voices. International Journal of Speech-Language Pathology. 15(5):484-491. doi: 10.3109/17549507.2012.744429

7. Roy N, Merrill RM,Gray SD, Smith EM (2005) Voice Disorders in the General Population: Prevalence, Risk Factors, and Occupational Impact. The Laryngoscoper 115(11):1988-1995. doi.org/10.1097/01.mlg.0000179174.32345.41

8. Anne Elise Richie (2013). The Professional Female Singer and Career Longevity: Reflections, Choices, and Challenges. The Florida State University College of Music, Florida State University.

9. Martha Elliott (2017) Singing Through Menopause: Reactions and Responses - Survey Results. Journal of Singing. 73(3):269-278.

10. Lortie CL, Rivard J, Thibeault M, et al. (2017)The moderating effect of frequent singing on voice aging. Journal of Voice. 31:112.e1-112.e12. doi:10.1016/ j.jvoice.2016.02.015.

11. Sundberg J (1995). Acoustic and psychoacoustic aspects of vocal vibrato. Vibrato, 35-62.

12. Reubold U, Harrington J, Kleber F (2010) Vocal aging effects on F0 and the first formant: A longitudinal analysis in adult speakers. Speech Communication. doi.org/10.1016/j.specom.2010.02.012

52(7-8):638-651.

13. Eichhorn JT, Kent RD, Austin D, Vorperian HK (2017) Effects of aging on vocal fundamental frequency and vowel formants in men and women. Journal of Voice. S0892-1997(17)30161-3. doi:10.1016/j.jvoice.2017.08.003

14. Das B, Mandal S, Mitra P, Basu A (2013) Effect of aging on speech features and phoneme recognition: a study on Bengali voicing vowels. International Journal of Speech Technology 16(1): 19-31.

15. Harrington J, Palethorpe S, Watson C. I (2007) Age-related changes in fundamental frequency and formants: a longitudinal study of four speakers. Proceedings of Interspeech. 2:1081-1084.

16. Steve An Xue, Grace Jianping Hao (2003) Changes in Human vocal tract due to ageing and the acoustic correlated of speech production: A pilot study. Journal of speech, Language, and Hearing Research. 46(3): 689 - 701. doi.org/10.1044/1092-4388(2003/054)

17. Indranil C, Hindol H, Sayani B, Suman K, Amitabha R (2011) An analytical study of age and gender effects on voice range profile in Bengali Adult speakers using phonetogram. International Journal of Phonosurgery and $\quad$ Laryngology. $65-70$. doi.org/10.5005/jp-journals-10023-1016.

18. Iseli M, Shue Y, Alwan A (2006) Age- and Gender-Dependent Analysis of Voice Source Characteristics. Proc. ICASSP. I-389 I-392. doi.org/10.1109/icassp.2006.1660039.

19. Lee SJ, Cho Y, Song JY, Lee D, Kim Y, Kim H (2015) Aging effect on Korean female voice: acoustic and perceptual examinations of breathiness. Folia Phoniatrica et Logopaedica. 67(6):300-307. doi.org/10.1159/000445290.

20. Tarafder KH, Datta PG, Tariq A (2012) The Aging Voice. BSMMU J 5(1):83-86

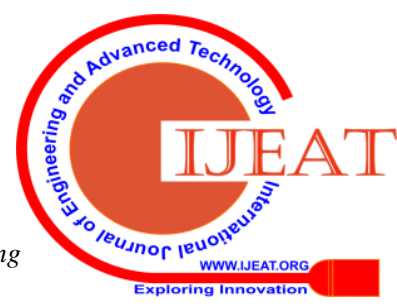


21. Verdonck-de Leeuw IM, Mahieu HF (2004) Vocal aging and the impact of daily life: A longitudinal study. Journal of Voice. 18(2):193-202. doi.org/10.1016/j.jvoice.2003.10.002.

22. R. Vipperla, S. Renals, and J. Frankel (2010) Ageing Voices: The Effect of Changes in Voice Parameters on ASR Performance. EURASIP J. Audio, Speech, and Music Proc. 1-10. doi.org/10.1155/2010/525783

23. Violet O Cox, Mark Selent (2015) Acoustic and Respiratory Measures as a Function of Age in the Male Voice. Journal of Phonetics \& Audiology. 1(1):1-7 doi:10.4172/2471-9455.1000105.

24. Arnold, A. (2001, January 01). Mangeshkar, Lata. Grove Music Online. Ed. Retrieved 24 Dec. 2018, from http://www.oxfordmusiconline.com/grovemusic/view/10.1093/gmo/9 781561592630.001.0001/omo-9781561592630-e-0000048965. doi.org/10.1093/gmo/9781561592630.article.48965.

25. Srivastava, Sanjay (2004) Voice, Gender and Space in Time of Five-Year Plans: The Idea of Lata Mangeshkar. Economic and Political Weekly. 39(20):2019-2028. URL: http://www.jstor.org/stable/4415027

26. Ashwini Deshpande (2004) Lata Mangeshkar: The Singer and the Voice. Economic and Political Weekly. 39(48):5179-5184 URL http://www.jstor.org/stable/4415842

27. Gregory J Sandell (1995) Roles of Spectral Centroid and other Factors in determining "Blended" instrument pairings in Orchestration. Music Perception: An interdisciplinary Journal. 13(2):209-246. doi.org/10.2307/40285694.

28. Sebastian S, Babu S, Oommen NE, Ballraj A (2012) Acoustic measurements of Geriatric Voice. J. Laryngol Voice Volume: 2,| Issue: 2, pp. 81-84. DOI: 10.4103/2230-9748.106984

29. Lortie CL, Thibeault M, Guitton MJ, et al. (2015) Effects of age on the amplitude, frequency and perceived quality of voice. Age (Omaha). 37:117. doi:10.1007/s11357-015-9854-1.

30. Kitch J, Oates J (1994) The perceptual features of vocal fatigue as self-reported by a group of actors and singers. Journal of Voice. 8(3):207-214. doi.org/10.1016/S0892-1997(05)80291-7.

31. Stemple JC, Stanley J, Lee L (1995) Objective measures of voice production in normal subjects following prolonged voice use. Journal of Voice. 9(2):127-133. doi.org/10.1016/s0892-1997(05)80245-0.

32. Damste' H, Reinders A, Tempelaars S (1982) Why should voices quiver? In: Hurme P, editor. Vox Humana, Institute of Finnish Language and Communication, University Jyvaskyla.

33. Ferrante, I (2011) Vibrato rate and extent in soprano voice: A survey on one century of singing. The Journal of the Acoustical Society of America. 130(3):1683-1688. https://doi.org/10.1121/1.3621017.

34. Hirano M, Hibi S, Hagino S (1995) Physiological aspects of vibrato. Vibrato.

35. Sundberg J, Niska-Thörnvik M (1998) Age and voice quality in professional singers. Logopedics, Phoniatrics, Vocology. 23(4):169-176. doi.org/10.1080/140154398434077.

36. Titze IR, Story B, Smith M, Long R (2002) A reflex resonance model of vocal vibrato. The Journal of the Acoustical Society of America. 111(5):2272-2282. https://doi.org/10.1121/1.1434945.

37. Lee Sang-Hyuk, Hee-Jun Kwon, Hyun-Jin Choi, Nam-Hun Lee, Sung-Jin Lee, SungMin Jin (2008) The Singer's Formant and Speaker's Ring Resonance: A Long-Term Average Spectrum Analysis. Clinical and Experimental Otorhinolaryngology 1(2):92-96. doi.org/10.3342/ceo.2008.1.2.92.

38. Omori K, Kacker A, Carroll LM.et al. (1996) Singing Power Ratio: Quantitative Evaluation of Singing Voice Quality. Journal of Voice. 10(3):228-235. doi: 10.1016/S0892-1997(96)80003-8

\section{AUTHORS PROFILE}

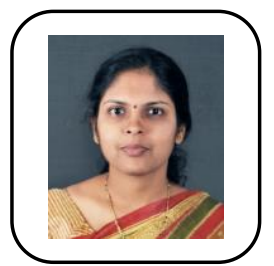

Ms. Deepali Loni, M.E (Electronics) from Walchand College of Engineering (2010) is pursuing her Ph.D. from Shivaji University, Kolhapur, in Signal Processing domain. She has more than 19 years of teaching experience at UG and 7 years at PG level for the courses in Electronics Engineering. Her specialization is in Digital signal Processing. She has done nine publications and presented more than ten papers in the international conferences. She has received 'Best Performance in Teaching' Award three times at the institute level. She has delivered several expert lectures at various institutes in the domain of signal processing.
Dr. Mrs. Shaila Subbaraman, Ph. D. from I.I.T., Bombay (1999) and

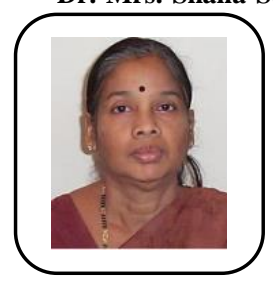
M. Tech. from I.I.Sc., Bangalore (1975) has a vast experience in industry in the capacity of R \& D engineer and Quality Assurance Manager in the field of manufacturing semiconductor devices and ICs. She also has more than 27 years of teaching experience at both UG and PG level for the courses in Electronics Engineering. She is a recognized $\mathrm{Ph}$ D guide of Shivaji University, Kolhapur and has guided and is guiding a couple of Ph D students. Her specialization is in Micro-electronics and VLSI Design. She has more than fifty publications to her credit. She retired as Dean Academics of autonomous Walchand College of Engineering, Sangli and currently she is working as Professor (PG) in the same college. Additionally she works as a NBA expert for evaluating engineering programs in India in accordance with Washington Accord. Recently she has been felicitated by "Pillars of Hindustani Society" award instituted by Chamber of Commerce, Mumbai for contribution to Higher Education in Western Maharashtra. 